\title{
Implementation of adomian polynomials in variational iteration method for solving volterra integral equations
}

\author{
Zainab Ayati ${ }^{1 *}$, Jafar Biazar $^{2}$, Sajedeh Zarei \\ ${ }^{1}$ Department of Engineering sciences, Faculty of Technology and Engineering East of Guilan, \\ University of Guilan, P.C. 44891-63157, Rudsar-Vajargah, Iran \\ ${ }^{2}$ Faculty of Mathematical Science, University of Guilan, Department of Applied Mathematics, \\ P.O. Box 41635-19141, P.C. 4193833697, Rasht, Iran \\ *Corresponding author E-mail: zainab.ayati@guilan.ac.ir
}

Copyright $\odot 2014$ Zainab Ayati et al. This is an open access article distributed under the Creative Commons Attribution License, which permits unrestricted use, distribution, and reproduction in any medium, provided the original work is properly cited.

\begin{abstract}
In this paper Adomian polynomials and Variational Iteration Method are employed for finding Volterra integral equations. The proposed technique involves Adomian Polynomials in the correction functional equation. The solutions have been found by suggested iterative scheme without any discretization, linearization, or restrictive assumptions, and it is quite efficient and is practically well suited. Two examples are given to verify the reliability and the efficiency of this method.
\end{abstract}

Keywords: Variational Iteration Method, Adomian Polynomials, Volterra Integral Equations, Functional Equation.

\section{Introduction}

Solving functional equations are important because they have the ability to model many phenomena in real world. So, researchers are interested in finding the best way to determine the solution of equations. He [1-10] developed the variational iteration (VIM) and homotopy perturbation methods (HPM) for solving linear, nonlinear, initial and boundary value problems. It is worth mentioning that the origin of variational iteration method can be traced back to Inokuti, Sekine and Mura [11], but the real potential of this technique was explored by Him, Chinese engineer. The basic motivation of this paper is to apply the variational iteration method coupled with Adomian Polynomials for finding the solution of Volterra integral equation. In this algorithm the correct functional is developed and the Lagrange multipliers are calculated optimally via variational theory. The use of Lagrange multipliers reduces the successive application of the integral operator and the cumbersome of huge computational work while it is still maintaining a very high level of accuracy. The developed algorithm takes full advantage of He's variational iteration and the Adomian decomposition methods. In following the concepts of methods are presented.

\section{Variational Iteration method}

To illustrate the basic concept of the technique, we consider the following general functional equation $L(u(x))+N(u(x))=g(x)$,

Where $L$ is a linear operator, $N$ is a nonlinear operator and $g(x)$ is the forcing term. According to variational iteration method, we can construct a correct functional as follows

$u_{n+1}(x)=u_{n}(x)+\int_{0}^{x} \lambda\left(L\left(u_{n}(s)\right)+N\left(\tilde{u}_{n}(s)\right)-g(s)\right) d s$,

Where $\lambda$ is a Lagrange multiplier, which can be identified optimally via variational iteration method? The subscripts $\mathrm{n}$ denote the $n$-th approximation, and $\tilde{u}_{n}$ is considered as a restricted variation. i. e. $\delta \tilde{u}_{n}=0$. Eq. (2) is called as a correction functional. 
The successive approximation $u_{n+1}, n \geq 0$ of the solution $u$ will be readily obtained. In this method, it is required first to determine the Lagrange multiplier $\lambda$ optimally upon using the determined Lagrange multiplier and any selective function $u_{0}$, consequently, the solution is given by $u=\lim _{n \rightarrow \infty} u_{n}$.

\section{Variational iteration method using adomian polynomials}

To illustrate the basic concept of the technique, let's consider the following general equation

$L[u(x)]+N[u(x)]=g(x)$,

Where $L$ is a linear operator, $N$ a nonlinear operator, and $g(x)$ is the source inhomogeneous term. According to section 2, we can construct a correctional functional as follows

$u_{n+1}(x)=u_{n}(x)+\int_{0}^{x} \lambda\left(L\left(u_{n}(s)\right)+N\left(\tilde{u}_{n}(s)\right)-g(s)\right) d s, \quad n \geq 0$.

Adomian considers the solutions as a power series say:

$V=\sum_{i=0}^{\infty} p^{i} v_{i}=v_{0}+p v_{1}+p^{2} v_{2}+p^{3} v_{3}+\ldots$

And the non-linear term as a series in terms of Adomian polynomials

$N(u)=\sum_{n=0}^{\infty} A_{n}\left(u_{0}, u_{1}, \ldots, u_{n}\right)$.

After determining $\lambda$, we substitute (5) and (6) in (4); integral part multiplied in $p$.

$$
\begin{aligned}
& \sum_{n=0}^{\infty} p^{n} v_{n}(x)=u_{0}(x)+p \int_{0}^{x} \lambda\left\{\sum_{n=0}^{\infty} p^{n}\left(L\left(v_{n}(s)\right)+N\left(\tilde{v_{n}}(s)\right)\right)-g(s)\right\} d s \\
& =u_{0}(x)+p \int_{0}^{x} \lambda\left\{\sum_{n=0}^{\infty} p^{n} L\left(v_{n}\right)+\sum_{n=0}^{\infty} p^{n} N\left(\tilde{v_{n}}\right)\right\} d s-p \int_{0}^{x} \lambda g(s) d s .
\end{aligned}
$$

The comparison of the same powers of $p$ gives solutions of various orders which are the variational iteration method using Adomian polynomials.

\section{Numerical applications}

In this section, we apply the variational iteration method using Adomian polynomials for solving Volterra integral equations.

Example 4.1: Consider the following nonlinear Volterra integral equation of the first kind,

$\int_{0}^{x} \cos (x-t) u(t) d t=x \sin x$,

The exact solution of the problem is

$u(x)=2 \sin x, 0<\mathrm{x}<1$.

By differentiation with respect to $x$ we have

$u(x)-\int_{0}^{x} \sin (x-t) u(t) d t=\sin x+x \cos x$,

And then

$u(x)=\sin x+x \cos x+\int_{0}^{x} \sin (x-t) u(t) d t$,

We changed the integral equation of the first kind to a second kind by differentiation.

The correction functional is given as the following form

$u_{n+1}(x)=u_{n}(x)+\int_{0}^{x} \lambda\left(u_{n}^{\prime}(s)-2 \cos (s)+s \sin (s)-\frac{d}{d s} \int_{0}^{S} \sin (s-t) u_{n}(t) d t\right) d s, n=0,1, \cdots$

Making the correction functional stationary, the Lagrange multiplier can easily be identified as $\lambda=-1$.

Applying the variational iteration method using polynomials Adomian, we obtain

$v_{0}+p v_{1}+p^{2} v_{2}+p^{3} v_{3}+\ldots=\sin x+x \cos x$

$+p \int_{0}^{x}(-1)\left(\begin{array}{c}v_{0}^{\prime}(s)+p v_{\mathbf{i}}^{\prime}(s)+p^{2} v_{2}^{\prime}(s)+\ldots-2 \cos (s)+s \sin (s) \\ -\frac{d}{d s} \int_{0}^{s}\left(\sin (s-t)\left(v_{0}(t)+p v_{1}(t)+p^{2} v_{2}(t)+\ldots\right) d t\right.\end{array}\right) d s$,

Comparing the coefficient of the same powers of $p$, consequently, the following approximants are obtained $p^{0}: v_{0}(x)=\sin x+x \cos x$,

$p^{1}: v_{1}(x)=\frac{1}{4} \sin x-\frac{1}{4} x \cos x+\frac{1}{4} x^{2} \sin x$, 


$$
\begin{aligned}
& p^{2}: v_{2}(x)=-\frac{1}{8} \sin x+\frac{1}{8} x \cos x-\frac{1}{4} x^{2} \sin x-\frac{1}{24} x^{3} \cos x, \\
& p^{3}: v_{3}(x)=\frac{5}{64} \sin x-\frac{5}{64} x \cos x+\frac{15}{64} x^{2} \sin x+\frac{7}{96} x^{3} \cos x-\frac{1}{192} x^{4} \sin x, \\
& \vdots
\end{aligned}
$$

The solution in a series form is given by

$$
u(x)=\lim _{p \rightarrow 1} \sum_{n=0}^{\infty} p v^{n} v_{n}=v_{0}+v_{1}+v_{2}+v_{3}+\mathrm{L}
$$

Table 4.1: Comparing the Numerical Result of Modified Method with the Exact Solution of Eq. (8).

\begin{tabular}{cccc}
\hline $\mathrm{x}$ & Exact solution & Series solution & Errors \\
\hline 0.0 & 0.0000000000 & 0.0000000000 & 0.0000000000 \\
0.2 & 0.3973386616 & 0.3973280356 & 0.0000106260 \\
0.4 & 0.7788366846 & 0.7785005224 & 0.0003361622 \\
0.6 & 1.1292849470 & 1.1267806570 & 0.0025042900 \\
0.8 & 1.4347121820 & 1.4244399360 & 0.0102722460 \\
1.0 & 1.6829419700 & 1.6526697270 & 0.0302722430 \\
\hline
\end{tabular}

Table 4.1 exhibits the exact solution and the series solution along with the errors obtained by using the modified method. It is obvious that the errors can be reduced further and higher accuracy can be obtained by evaluating more components of $\mathrm{u}(\mathrm{x})$.

Example 4.2: Consider the following nonlinear Volterra integral equation,

$u(x)=x-\int_{0}^{x}(x-t) u(t) d t$,

The exact solution of the problem is

$u(x)=\sin x, 0<\mathrm{x}<1$.

The correction functional is given as follows

$u_{n+1}(x)=u_{n}(x)+\int_{0}^{x} \lambda\left(u_{n}^{\prime}(s)-1-\frac{d}{d s} \int_{0}^{S}(s-t) u_{n}(t) d t\right) d s, n=0,1, \cdots$

Making the correction functional stationary, the Lagrange multiplier can easily be identified as $\lambda=-1$.

Applying the variational iteration method using polynomials, we obtain

$v_{0}+p v_{1}+p^{2} v_{2}+p^{3} v_{3}+\ldots=x$

$+p \int_{0}^{x}(-1)\left(v_{0}^{\prime}(s)+p v_{\mathbf{i}}^{\prime}(s)+p^{2} v_{2}^{\prime}(s)+\ldots-1+\frac{d}{d s} \int_{0}^{s}(s-t)\left(v_{0}(t)+p v_{1}(t)+p^{2} v_{2}(t)+\ldots\right) d t\right) d s$,

Comparing the coefficient of the same powers of $p$, consequently, the following approximants are obtained $p^{0}: v_{0}(x)=x$,

$p^{1}: v_{1}(x)=-\frac{1}{6} x^{3}=-\frac{1}{3 !} x^{3}$,

$p^{2}: v_{2}(x)=\frac{1}{6} x^{3}+\frac{1}{120} x^{5}=\frac{1}{3 !} x^{3}+\frac{1}{5 !} x^{5}$,

$p^{3}: v_{3}(x)=-\frac{1}{6} x^{3}-\frac{1}{60} x^{5}-\frac{1}{5040} x^{7}=-\frac{1}{3 !} x^{3}-\frac{2}{5 !} x^{5}-\frac{1}{7 !} x^{7}$,

The solution in a series form is given by

$u(x)=\lim _{p \rightarrow 1} \sum_{n=0}^{\infty} p^{n} v_{n}=v_{0}+v_{1}+v_{2}+v_{3}+\ldots$

Table 4.2: Comparing the Numerical Result of Modified Method with the Exact Solution of Eq. (12).

\begin{tabular}{cccc}
\hline $\mathrm{x}$ & Exact solution & Series solution & Errors \\
\hline 0.0 & 0.0000000000 & 0.0000000000 & 0.0000000000 \\
0.2 & 0.1986693308 & 0.1986639975 & 0.0000053333 \\
0.4 & 0.3894183423 & 0.3892476749 & 0.0001706674 \\
0.6 & 0.5646424734 & 0.5633464457 & 0.0054617011 \\
0.8 & 0.7173560909 & 0.7118943898 & 0.0166693975 \\
1.0 & 0.8414709748 & 0.8248015873 & \\
\hline
\end{tabular}

Table 4.2 exhibits the exact solution and the series solution along with the errors obtained by using the modified method. It is obvious that the errors can be reduced further and higher accuracy can be obtained by evaluating more components of $\mathrm{u}(\mathrm{x})$. 


\section{Conclusion}

In this paper, the modified variational iteration method has been developed for solving Volterra Integral Equations. The method is applied in a direct way without using linearization, transformation, discretization or restrictive assumptions. The proposed method is successfully implemented by using the initial conditions.

It may be concluded that the variational iteration method using polynomials is very powerful and efficient in finding the analytical solutions for a wide class of boundary value problems. The calculations are obtained with the help of Maple 15.

\section{References}

[1] J. H. He, "Some asymptotic methods for strongly nonlinear equation", Int. J. Mod. Phys., 10 (20) (2006) 1144-1199.

[2] J. H. He, "Homotopy perturbation technique", Comput. Math. Appl. Mech, Energy, 178 (1999) 178-257.

[3] J. H. He, "Homotopy perturbation method for solving boundary value problems", Phys. Lett A, 350 (2006) 87-88. http://dx.doi.org/10.1016/j.physleta.2005.10.005.

[4] J. H. He, "Comparison of homotopy perturbation method and homotopy analysis method", Appl. Math. Comput, 156 (2004) 527-539. http://dx.doi.org/10.1016/j.amc.2003.08.008.

[5] J. H. He, "Homotopy perturbation method for bifurcation of nonlinear problems", Int. J. Nonlin. Sci Num. Simul, 6 (2) (2005) $207-208$. http://dx.doi.org/10.1515/IJNSNS.2005.6.2.207.

[6] J. H. He, "Variational iteration method. A kind of non-linear analytical technique, some examples", Internat. J. Nonlinear Mech, 34 (4) (1999) 699-708. http://dx.doi.org/10.1016/S0020-7462 (98)00048-1.

[7] J. H. He, "Variational iteration method for autonomous ordinary differential systems", Appl. Math. Comput., 114 (2-3) (2000) 115-123. http://dx.doi.org/10.1016/S0096-3003 (99)00104-6.

[8] J. H. He, X. H. Wu, "Construction of solitary solution and compaction-like solution by variational iteration method", Chaos, Solitons and Fractals, 29 (1) (226) 108-113.

[9] J. H. He, "Variational iteration method- Some recent results and new interpretations", J. Comput. Appl. Math, 207 (2007) 3-17. http://dx.doi.org/10.1016/j.cam.2006.07.009.

[10] J. H. He, X. H. Wu, "Variational iteration method: New development and applications", Comput. Math. Appl, 54 (2007) 881-894. http://dx.doi.org/10.1016/j.camwa.2006.12.083.

[11] M. Inokuti, H. Sekine,T. Mura, "General use of the Lagrange multiplier in non-linear mathematical physics, in: S. Nemat-Nasser (Ed.),Variational Method in the Mechanics of Solids", Pergamon Press, Oxford, 1978, 156-162. 